\title{
TEOR DE COMPOSTOS FENÓLICOS E ATIVIDADE ANTIOXIDANTE EM DIFERENTES GENÓTIPOS DE AMOREIRA-PRETA(Rubus sp.) ${ }^{1}$
}

\author{
MÁRCIA VIZZOTTO², MARIA DO CARMO BASSOLS RASEIRA², \\ MARINA COUTO PEREIRA ${ }^{3}$ MARIANA DA ROSA FETTER ${ }^{4}$
}

RESUMO-Tendo em vista que alguns compostos fenólicos encontrados em amora-preta são benéficos para a saúde humana, este estudo foi conduzido com o propósito de quantificar os teores de compostos fenólicos totais e a atividade antioxidante em diferentes genótipos produzidos por 10 seleções e 4 cultivares de amoreira-preta com ou sem espinhos. Quanto aos resultados, pode-se observar que, dentre as seleções e cultivares de amoreira-preta com espinho, a seleção S16/96 apresentou o maior teor de compostos fenólicos e atividade antioxidante. Dentre as seleções sem espinho, a S17/01 e a S02/96 apresentaram os maiores valores, não diferindo da S12/01 para a atividade antioxidante. Não houve diferença estatística para os teores de compostos fenólicos totais e atividade antioxidante, quando comparadas entre si as seleções e as cultivares de amoreira-preta com espinhos. A correlação entre o teor de compostos fenólicos totais e a atividade antioxidante nas amoras-pretas estudadas é baixa, indicando presença de outros fitoquímicos e/ou vitaminas que podem influenciar o poder antioxidante.

Termos para indexação: compostos bioativos, cultivares e seleções, saúde.

\section{PHENOLIC CONTENT AND ANTIOXIDANT ACTIVITY OF DIFFERENT GENOTYPES OF BLACKBERRY (Rubus sp.)}

\begin{abstract}
Considering that some phenolic compounds found in blackberry are beneficial to human health, this study was conducted in order to quantify the levels of total phenolics and antioxidant activity in genotypes produced by different 10 cultivars and 4 selections of thorn and thornless blackberry plants. Concerning the results, it can be observed that among the thorn blackberry selections and cultivars, the selection S16/96 had the highest phenolic content and antioxidant activity. Among the thornless blackberry selections, the selections S17/01 and S02/96 showed higher values; however, it did not differ from selection S12/01 regarding to antioxidant activity. There was no statistical difference in the levels of total phenolics and antioxidant activity when compared with each other selections and cultivars of thorn blackberries. The correlation between total phenolic content and antioxidant activity in this blackberries study is weak indicating the presence of other phytochemicals and vitamins that may influence the antioxidant power.
\end{abstract}

Index terms: bioactive compounds, cultivars and selections, health.

\section{INTRODUÇÃO}

Embora existam espécies nativas no Brasil, a amoreira-preta só começou a ser pesquisada em 1972 pela Embrapa Clima Temperado, sendo a primeira coleção implantada em 1974 no município de Canguçu-RS (HEIDEN et al., 2004). Estima-se que a área de produção de amora-preta no Brasil seja de 150 ha, estendendo-se do Espírito Santo, Rio de Janeiro e sul de Minas Gerais, ao sul do Rio Grande do Sul (ANTUNES et al., 2007).
A amoreira-preta ("blackberry”) pertence ao gênero Rubus que, segundo Ying et al. (1990), contém, aproximadamente, 740 espécies, divididas, segundo alguns autores, em 12 subgêneros ou, segundo outros, em 15 subgêneros (DAUBENY, 1996). É uma espécie arbustiva de porte ereto ou rasteiro, que produz frutos agregados, com cerca de 4 a 7 gramas, coloração negra e sabor ácido a doce-ácido. Apresenta espinhos em suas principais cultivares comerciais, o que exige do operador da colheita muito cuidado com sua integridade física e com a qualidade do fruto

${ }^{1}$ (Trabalho 163-11). Recebido em: 27-05-2011. Aceito para publicação em: 28-08-2012.

2Ph.D., Embrapa Clima Temperado, BR 392, KM 78, C. P. 403, CEP 96010-971, Pelotas-RS. E-mail: marcia.vizzotto@cpact.embrapa.br; maria.bassols@cpact.embrapa.br

${ }^{3}$ Nutricionista, Doutoranda no Programa de Pós-graduação em Ciência e Tecnologia Agroindustrial, Universidade Federal de Pelotas, Pelotas-RS, Brasil. E-mail: marinacoutopereira@hotmail.com

${ }^{4}$ Graduanda em Ciências Biológicas, Universidade Católica de Pelotas, Pelotas-RS, Brasil. E-mail: marianafetter@hotmail.com 
(FACCHINELLO et al., 1994). Porém, trabalhos de melhoramento genético desta frutífera vêm sendo realizados visando ao desenvolvimento de cultivares sem espinho, com alta produtividade, maior peso de frutos e resistência pós-colheita (ANTUNES, 2002). Entre as principais cultivares exploradas no Rio Grande do Sul e lançadas pela Embrapa Clima Temperado, estão a 'Tupy' e a 'Guarani', ambas com espinhos (SANTOS; RASEIRA, 1988) e 'Xavante' (MOORE et al., 2004) sem espinho.

A amora-preta in natura é altamente nutritiva, apresentando elevado conteúdo de minerais, vitaminas B, A e cálcio. Essa fruta pode ser consumida de outras formas, como geleias, suco, sorvete e iogurtes (POLING, 1996). Uma série de funções e constituintes químicos é relatada na literatura internacional relacionados às qualidades da amora-preta, estando, entre eles, o ácido elágico, um composto fenólico que possui funções antioxidante, anti-mutagênica, anticancerígena e além de ser um potente inibidor da indução química do câncer (WANG et al., 1994; MAAS et al.,1991). Entretanto, de acordo com Sellapan et al. (2002) e Jacques et al. (2009), variações no conteúdo total de compostos fenólicos parecem ser frequentes entre as diferentes cultivares.

Dessa forma, este estudo foi conduzido com o propósito de quantificar os compostos fenólicos totais e a atividade antioxidante de frutas provenientes de 10 seleções (com e sem espinho) e quatro cultivares com espinhos de amoreira-preta cultivadas na região Sul do Brasil.

\section{MATERIAL E MÉTODOS}

Preparo de Amostras: As amoras-preta foram colhidas na safra de 2006/2007 e armazenadas em freezer a $-18^{\circ} \mathrm{C}$ até o momento das análises (em torno de um mês de armazenamento), quando foram homogeneizadas visualmente por tamanho e cor. Para cada ensaio,foram utilizadas 100 frutas. Destas frutas, foi retirada, para as análises, uma amostra de $5 g$ composta apenas pelas suas porções equatoriais, após cortá-las manualmente com faca de cozinha. As amostras foram preparadas em triplicatas, trituradas em presença de etanol acidificado $(0,01 \%$ de $\mathrm{HCl})$, em homogeneizador do tipo ultraturrax, dimensionado para amostras pequenas, e centrifugadas em centrífuga refrigerada com temperatura em torno de $4^{\circ} \mathrm{C}$ a $15.000 \mathrm{rpm}$. O sobrenadante foi retido para as análises de compostos fenólicos totais e atividade antioxidante.

Foram avaliados os frutos provenientes das seguintes seleções de amoreira-preta: S16/96, S21/96, SBlack97, S787 americana, S03/01 (com espinho) e S02/96, S12/01, S14/96, S14/01, e S17/01 (sem espinho). As cultivares de amoreira-preta avaliadas foram todas provenientes de plantas com espinho, tais como Cheroke, Comanche, Guarani e Tupy.

Análise de Fenólicos Totais: A metodologia utilizada para determinação de compostos fenólicos totais foi adaptada de Swain e Hillis (1959). Para cada tubo de ensaio, foram pipetados $250 \mathrm{uL}$ da amostra, adicionados $4 \mathrm{~mL}$ de água ultrapura e $250 \mathrm{uL}$ do reagente Folin-Ciocalteau $(0,25 \mathrm{~N})$, os quais foram agitados e mantidos por 3 minutos para reagir. Após este tempo, adicionaram-se 500 uL de carbonato de sódio (1N), e os tubos novamente foram agitados e mantidos por 2 horas para reagir. O espectrofotômetro foi zerado com o controle (branco), e foram feitas as leituras da absorbância no comprimento de onda de $725 \mathrm{~nm}$.

Análise de Atividade Antioxidante: A metodologia utilizada para a determinação da atividade total foi adaptada de Brand-Williams et al. (1995). Em resumo, foram pipetados $200 \mathrm{uL}$ de amostra e misturados com 3.800 uL de DPPH (diluído em metanol) em tubos de $15 \mathrm{~mL}$ com tampa. Os tubos foram agitados e deixados para reagir por 24 horas. Para a leitura no espectrofotômetro, usou-se o metanol para zerar o equipamento, e a absorbância foi medida no comprimento de onda de $525 \mathrm{~nm}$.

Todas as análises foram feitas em quadruplicata. Os resultados foram avaliados através da análise de variância ANOVA e pelo teste de Tukey, ambos a 5\% de significância, utilizando-se do Programa Statistica 7.0.

\section{RESULTADOS E DISCUSSÃO}

Comparando os genótipos de amoreiras-pretas com e sem espinhos não se observou diferença significativa no que se refere ao teor de compostos fenólicos nem mesmo em atividade antioxidante (Tabela 1).

Deve-se ressaltar que, no Brasil, ocorrem cinco espécies nativas de amoras, as quais produzem frutos pequenos e com coloração branca, rosa, vermelha ou preta, sendo que nenhuma destas espécies brasileiras foi domesticada. As cultivares de amoreiras-pretas exploradas comercialmente no País são o resultado de introduções, hibridações e seleções de cultivares americanas (RASEIRA et al., 2008). As cultivares sem espinhos de amoreira-preta foram originadas de cruzamentos interespecíficos com espécies como a Rubus ulmifolius, nas quais havia plantas sem espinhos, provavelmente devido à mutação natural. Teoricamente, a planta sem espinhos, por estar desprovida de proteção contra pragas, como insetos, 
deveria sintetizar mais compostos secundários como forma de defesa, fato não comprovado neste trabalho. A tendência dos programas de melhoramento é buscar plantas sem espinhos para facilitar o manejo do pomar e a colheita das frutas, preferencialmente, sem perder a qualidade. Outro motivo é para facilitar a colheita mecanizada, pois as amoreiras-pretas com espinhos podem ficar com estes encravados no fruto durante a colheita mecanizada, trazendo uma série de problemas ao consumidor e aos produtores (DU et al., 2010).

Pantelidis et al. (2007) observaram que os teores de antocianinas totais, compostos fenólicos e a atividade antioxidante foram inferiores em cultivares de amoreira-preta com espinhos quando comparadas a cultivares sem espinho. Du et al. (2010) também encontraram variações significativas na concentração de determinadas substâncias, dando, desta forma, características específicas a cada genótipo. Esta pode ser uma explicação para a síntese de compostos secundários em amoreiras-pretas com e sem espinho. Os teores totais de compostos fenólicos podem ser semelhantes, mas a análise individual dos compostos pode revelar que alguns tipos de compostos fenólicos podem ser sintetizados em maiores ou menores concentrações em uma planta do que em outra.

Analisando somente as amoras-pretas produzidas em plantas com espinhos, a média do grupo de cultivares não diferiu da média do grupo de seleções, quanto ao teor de compostos fenólicos totais e a atividade antioxidante (Tabela 2). Provavelmente, porque as cultivares estudadas fazem parte da base genética das seleções que foram avaliadas, sendo assim, não há grande variação nos teores de compostos fenólicos totais nem na atividade antioxidante.

Entre as cultivares e seleções de amoras-pretas produzidas em plantas que apresentavam espinhos, a seleção S16/96 e a S14/96, e as cultivares Comanche e Guarani apresentaram os maiores teores de compostos fenólicos totais. Já a atividade antioxidante foi superior nas seleções S16/96 e S787 americana, não diferindo da cultivar Comanche (Tabela 3). As cultivares Cheroke e Comanche foram introduzidas dos Estados Unidos, e as cultivares Tupy e Guarani foram lançadas pela Embrapa Clima Temperado (RASEIRA et al., 1984; RASEIRA et al., 1992), entretanto estas apresentam ancestrais americanos em sua genealogia.

As propriedades antioxidantes estão muito relacionadas ao tipo de fruta, ou seja, espécie ou cultivares dentro da mesma espécie, e às condições de cultivo das plantas, como ambiente e técnicas de manejo (SCALZO et al., 2005), além do grau de maturidade na colheita, sendo que, quanto mais maduras as frutas, menor o teor de compostos fenólicos totais e a atividade antioxidante (WANG; LIN, 2000;
SIRIWOHARN et al., 2004; CASTREJON et al., 2008). Variação nos teores de compostos fenólicos em amora-preta é comumente observada. Moyer et al. (2002), analisando 37 genótipos de amoreira-preta, conduzidos em um mesmo local, observaram variação de mais de $100 \%$ nos teores de compostos fenólicos e da atividade antioxidante; em adição, comparando-se frutos da mesma cultivar, produzidos em diferentes locais, foi observado que o fator ambiente está altamente relacionado ao teor de compostos bioativos sintetizados (REYS-CARMONA et al., 2005; OLIVEIRA et al., 2009).

Em relação às seleções estudadas que não apresentavam espinhos, a seleção S17/01 apresentou um teor de compostos fenólicos superior às demais, não diferindo da S02/96. Quanto à atividade antioxidante, as seleções S02/96, a S12/01 e a S17/01 mostraram-se superiores quando comparadas com a S14/01 (Tabela 4). Estas seleções foram obtidas através de cruzamentos de genótipos sem sementes, realizados na Universidade de Arkansas. A base genética de origem deste material não está estabelecida; no entanto, pelos resultados apresentados, pode-se observar que existe variação entre as seleções tanto para o teor de compostos fenólicos, como para a atividade antioxidante. Mesmo comparando cultivares de amoreira-preta sem espinhos, pode-se observar que existe variação nos teores de compostos fenólicos totais, antocianinas e atividade antioxidante (WANG; LIN, 2000; MOYER et al., 2002; PANTEDELIS et al., 2007; KOCA; KARADENIZ, 2009).

Não houve boa correlação entre o conteúdo de compostos fenólicos e atividade antioxidante em amoras-pretas (Figura 1). A correlação entre o teor de compostos fenólicos totais e a atividade antioxidante ainda é bastante controversa. Em várias frutas e hortaliças, é possível estabelecer boa correlação entre estas duas variáveis; no entanto, em alguns casos, essa correlação apresenta-se baixa. Isto pode acontecer devido à presença de vários outros fitoquímicos, como as antocianinas ou vitaminas que podem influenciar na atividade antioxidante. Em diversos estudos com amora-preta, foi encontrada alta correlação linear positiva entre compostos fenólicos totais e a atividade antioxidante (WANG; LIN, 2000; MOYER et al., 2002). Seguindo o mesmo padrão, mirtilos produzidos na Turquia apresentaram correlação linear entre a atividade antioxidante, determinada pelo método FRAP, e o teor de compostos fenólicos totais de $\mathrm{r}=0.981$ (KOCA; KARADENIZ, 2009). Em amora-preta, sabe-se que a contribuição da vitamina $C$ não é tão importante para a atividade antioxidante como é no caso da framboesa e das frutas cítricas, como os limões e as laranjas (ATALA et al., 2009). 
TABELA 1 - Compostos fenólicos totais e atividade antioxidante em amora-preta provenientes de plantas com espinhos vs sem espinhos. Embrapa Clima Temperado, Pelotas-RS, 2010.

\begin{tabular}{lll}
\hline Grupos & Compostos fenólicos $^{1}$ & Atividade antioxidante $^{2}$ \\
\hline Sem espinho & $870,71 \pm 28,42 \mathrm{a}$ & $10245,96 \pm 375,05 \mathrm{a}$ \\
Com espinho & $839,24 \pm 23,83 \mathrm{a}$ & $9910,53 \pm 291,31 \mathrm{a}$ \\
\hline
\end{tabular}

Dados são médias dos genótipos do grupo \pm erro-padrão. Letras iguais na mesma coluna significa que não diferem entre si pelo teste de Tukey a $5 \%$. ${ }^{1}$ Compostos fenólicos totais expressos em mg do equivalente ácido clorogênico $100 \mathrm{~g}^{-1}$ amostra fresca. ${ }^{2}$ Atividade antioxidante total expressa em $\mu \mathrm{g}$ equivalente trolox $\mathrm{g}^{-1}$ amostra fresca.

TABELA 2- Compostos fenólicos totais e atividade antioxidante de seleções e cultivares de amoreira-preta com espinhos. Embrapa Clima Temperado, Pelotas-RS, 2010.

\begin{tabular}{lll}
\hline Com espinhos & Compostos fenólicos & Atividade antioxidante \\
\hline Cultivares & $816,24 \pm 34,70 \mathrm{a}$ & $9511,89 \pm 342,49 \mathrm{a}$ \\
Seleções & $854,57 \pm 32,57 \mathrm{a}$ & $10176,29 \pm 424,61 \mathrm{a}$ \\
\hline
\end{tabular}

Dados são médias dos genótipos do grupo \pm erro-padrão. Letras iguais na mesma coluna significa que não diferem entre si pelo teste de Tukey a 5\%. ${ }^{1}$ Compostos fenólicos totais expressos em mg do equivalente ácido clorogênico $100 \mathrm{~g}^{-1}$ amostra fresca. ${ }^{2}$ Atividade antioxidante total expressa em $\mu \mathrm{g}$ equivalente trolox $\mathrm{g}^{-1}$ amostra fresca.

TABELA 3- Compostos fenólicos totais e atividade antioxidante de seleções e cultivares de amoreira-preta com espinhos, que fazem parte do programa de melhoramento genético da Embrapa Clima Temperado. Embrapa Clima Temperado, Pelotas-RS, 2010.

\begin{tabular}{lll}
\hline Seleções/Cultivares & Compostos fenólicos $^{1}$ & Atividade antioxidante $^{2}$ \\
\hline S14/96 & $921,0 \pm 43,3 \mathrm{ab}$ & $10568,5 \pm 994,9 \mathrm{bc}$ \\
S16/96 & $1077,0 \pm 47,1 \mathrm{a}$ & $12604,8 \pm 898,3 \mathrm{a}$ \\
S21/96 & $819,2 \pm 86,1 \mathrm{bcd}$ & $9901,9 \pm 662,0 \mathrm{bcd}$ \\
SBlack97 & $699,9 \pm 64,9 \mathrm{~cd}$ & $7090,6 \pm 148,8 \mathrm{e}$ \\
S787 americana & $832,1 \pm 108,4 \mathrm{bcd}$ & $10735,2 \pm 951,4 \mathrm{ab}$ \\
S03/01 & $778,2 \pm 81,9 \mathrm{bcd}$ & $10156,8 \pm 693,7 \mathrm{bcd}$ \\
CHEROKE & $660,4 \pm 10,6 \mathrm{~d}$ & $8417,3 \pm 798,4 \mathrm{de}$ \\
COMANCHE & $901,9 \pm 141,1 \mathrm{abc}$ & $10915,5 \pm 678,2 \mathrm{ab}$ \\
GUARANI & $902,2 \pm 2,6 \mathrm{abc}$ & $10103,5 \pm 344,6 \mathrm{bcd}$ \\
TUPY & $800,4 \pm 23,4 \mathrm{bcd}$ & $8611,1 \pm 175,8 \mathrm{cde}$ \\
\hline
\end{tabular}

Dados são médias de três repetições \pm desvio-padrão. Letras iguais na mesma coluna significa que não diferem entre si pelo teste de Tukey a $5 \%$. ${ }^{1}$ Compostos fenólicos totais expressos em mg do equivalente ácido clorogênico $100 \mathrm{~g}^{-1}$ amostra fresca. ${ }^{2}$ Atividade antioxidante total expressa em $\mu \mathrm{g}$ equivalente trolox $\mathrm{g}^{-1}$ amostra fresca.

TABELA 4 - Compostos fenólicos totais e atividade antioxidante de seleções de amoreira-preta sem espinhos, que fazem parte do programa de melhoramento da Embrapa Clima Temperado. Embrapa Clima Temperado, Pelotas-RS, 2010.

\begin{tabular}{lll}
\hline Seleções & Compostos fenólicos $^{1}$ & Atividade antioxidante $^{2}$ \\
\hline S02/96 & $911,1 \pm 78,2 \mathrm{ab}$ & $11289,4 \pm 890,4$ a \\
S12/01 & $818,6 \pm 56,5 \mathrm{~b}$ & $11032,6 \pm 464,8 \mathrm{a}$ \\
$\mathrm{S} 14 / 01$ & $770,4 \pm 10,2 \mathrm{~b}$ & $8320,8 \pm 90,3 \mathrm{~b}$ \\
S17/01 & $982,7 \pm 58,7 \mathrm{a}$ & $10341,1 \pm 364,1$ a \\
\hline
\end{tabular}

Dados são médias de três repetições \pm desvio-padrão. Letras iguais na mesma coluna significa que não diferem entre si pelo teste de Tukey a $5 \%$. ${ }^{1}$ Compostos fenólicos totais expressos em mg do equivalente ácido clorogênico $100 \mathrm{~g}^{-1}$ amostra fresca. ${ }^{2}$ Atividade antioxidante total expressa em $\mu \mathrm{g}$ equivalente trolox $\mathrm{g}^{-1}$ amostra fresca. 


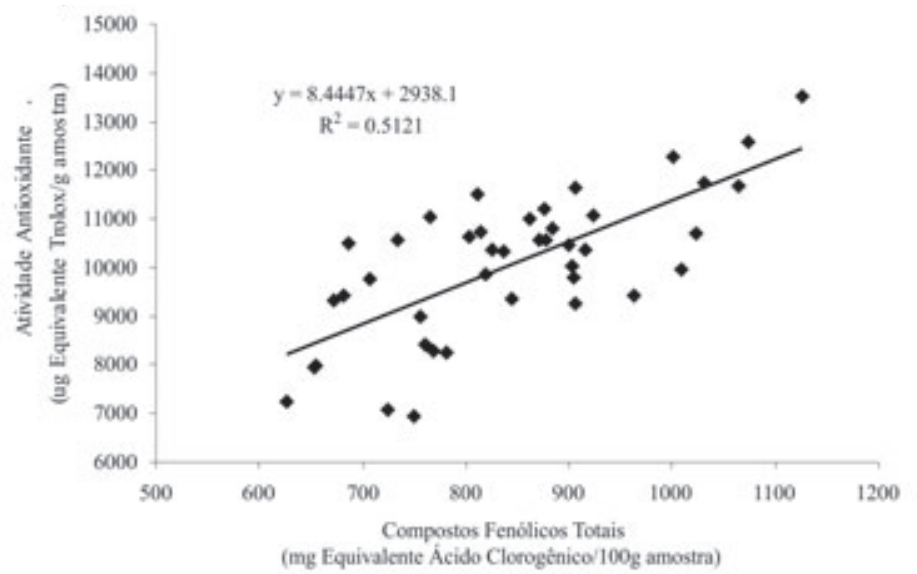

FIGURA 1 - Correlação entre compostos fenólicos totais e atividade antioxidante nas seleções e cultivares de amoras-pretas estudadas. Embrapa Clima Temperado, Pelotas-RS, 2010.

\section{CONCLUSÕES}

1-Dentre as seleções e cultivares de amorapreta, com espinho, a seleção S16/96 apresenta o maior teor de compostos fenólicos e atividade antioxidante, não diferindo da 'Comanche', S14/96, 'Guarani’ e S787 americana.

2-Dentre as seleções de amora-preta, sem espinho, a S17/01 e a S02/96 apresentam os maiores teores de compostos fenólicos e atividade antioxidante, não diferindo da S12/01 para atividade antioxidante.

3-Comparando os grupos de amora-preta com e sem espinhos, não se observa diferença para os teores de compostos fenólicos totais nem para a atividade antioxidante.

\section{REFERÊNCIAS}

ANTUNES, L. E. C. Amora - Preta: nova opção de cultivo no Brasil. Ciência Rural, Santa Maria, v. 32, n. 1, p.151-158, 2002.

ANTUNES, L. E. C.; TREVISAN, R.; PEREIRA, I. dos S. Produção de Amora-preta. In: SEMINÁRIO BRASILEIRO SOBRE PEQUENAS FRUTAS, 4., 2007, Vacaria. Anais... Vacaria: Embrapa Uva e Vinho, p. 65-71, 2007. (Documentos, 59).

ATALA, E.; VÁSQUEZ, L.; SPEISKY, H.; LISSI, E.; LÓPEZ-ALARCÓN, C. Ascorbic acid contribution to ORAC values in berry extracts: An evaluation by the ORAC-pyrogallol red methodology. Food Chemistry, Washington, v. 113, p. 331-335, 2009.
BRAND-WILLIAMS, W.; CUVELIER, M. E.; BERSET, C. Use of a free radical method to evaluate antioxidant activity. Lebensmittel-Wissenschaft und Technologie, London, v. 28, p. 25-30, 1995.

CASTREJON, A. D. R.; EICHHOLZ, I.; ROHN, S.; KROH, L. W.; HUYSKENS-KEIL, S. Phenolic profile and antioxidant activity of highbush blueberry (Vaccinium corymbosum L.) during fruit maturation and ripening. Food Chemistry, Washington, v. 109, p. 564-572, 2008.

DAUBENY, H. A. Brambles. In: MOORE, J. N.; JANICK, J. (Ed.). Fruit breeding. London: John Willey, 1996. v. 2, p. 109-190.

DU, X.; FINN, C. E. ; QIAN, M. C. Volatile composition and odour-activity value of thornless 'Black Diamond' and 'Marion' blackberries. Food Chemistry, Washington, v. 119, p. 1.127-1.134, 2010 .

FACCHINELLO, J. C.; HOFFMANN, A.; SANTOS, A. M. dos. Amoreira-preta, framboesa e mirtilo: pequenos frutos para o sul do Brasil. In: CONGRESSO BRASILEIRO DE FRUTICULTURA, 13., 1994, Salvador. Anais... Vitória da Conquista: Sociedade Brasileira de Fruticultura, 1994. v. 3, p. 989-990.

HEIDEN, G.; BARBIERI, R. L. ; GARRASTAZÚ, M. C.; GARCIA, L. O.; MAIA, M. B. Ocorrência de espécies nativas de amora no Rio Grande do Sul. In: SIMPÓSIO NACIONAL DO MORANGO, 2.; ENCONTRO DE PEQUENAS FRUTAS E FRUTAS NATIVAS DO MERCOSUL, 1., 2004, Pelotas. Resumos... Pelotas: Embrapa Clima Temperado, 2004. p. 345-352. (Documentos, 123). 
JACQUES, A. C.; PERTUZATTI, P. B.; BARCIA, M. T.; ZAMBIAZI, R. C. Nota científica: Compostos bioativos em pequenas frutas cultivadas na região sul do Estado do Rio Grande do Sul. Brazilian Journal of Food Technology, Campinas, v. 12, n. 2, p. 123127, 2009.

KOCA, I.; KARADENIZ, B. Antioxidant properties of blackberry and blueberry fruits grown in the Black Sea Region of Turkey. Scientia Horticulturae, Washington, v. 121, p. 447-450, 2009.

MAAS, J. L.; GALLETTA, G. J.; STONER, G. D. Ellagic acid, an anticarciogen in fruits, especially in strawberry: a review. HortScience, Alexandria, v. 26, n. 1, p. 10-14. 1991.

MOYER, R. A.; HUMMER, K. E.; FINN, C. E.; FREI, B.; WROLSTAD, R. E. Anthocyanins, phenolics, and antioxidant capacity in diverse small fruits: Vaccinium, Rubus, and Ribes. Journal of Agriculture and Food Chemistry, Washington, v. 50, p. 519-525, 2002.

OLIVEIRA, A. C. de; VALENTIM I, B.; GOULART, M. O. F.; SILVA, I. C.; BECHARA, E. J. H.; TREVISAN, M. T. S. Fontes vegetais naturais de antioxidantes. Química Nova, São Paulo, v. 32, n. 3, p.689-702, 2009.

PANTELIDIS, G. E.; VASILAKAKIS, M.; MANGANARIS, G. A.; DIAMANTIDIS, G. R. Antioxidant capacity, phenol, anthocyanin and ascorbic acid contents in raspberries, blackberries, red currants, gooseberries and Cornelian cherries. Food Chemistry, Washington, v. 102, p. 777-783, 2007.

POLING, E. B. Blackberries. Journal of Small Fruit and Viticulture, Beltsville, v. 14, n. 1-2, p. 38-69. 1996.

RASEIRA, A.; SANTOS, A. M. dos; RASEIRA, M. do C. B. Caingangue, nova cultivar de amoreira-preta para consumo ‘in natura'. HortiSul, Pelotas, v. 2, n. 3, p. 11-12, 1992.

3RASEIRA, M. do C. B.; SANTOS, A. M. dos; BARBIERI, R. L. Sistema de Produção da amoreira-preta: classificação botânica, origem e cultivares. Pelotas: Embrapa Clima Temperado, 2008. (Sistemas de Produção)
RASEIRA, M. do C. B.; SANTOS, A. M. dos; MADAIL, J. C. M. Amora preta: cultivo e utilização. Pelotas : EMBRAPA. CNPFT, 1984. 20p. (Circular Técnica, 11).

REYES-CARMONA, J.; YOUSEF, G. G.; MARTÍNEZ-PENICHE, R. A.; LILA, M. A. Antioxidant capacity of fruit extracts of blackberry (Rubus sp.) produced in different climatic regions. Journal of Food Science, Chicago, v. 70, n. 7, p. 497-503, 2005.

SANTOS, A. M.; RASEIRA, M. C. B. Lançamento de cultivares de amora-preta. Pelotas: EMBRAPACNPFT, 1988. (Informativo, 23).

SCALZO, J.; POLITI, A.; PELLEGRINI, N.; MEZZETTI, B.; BATTINO, M. Plant genotype affects total antioxidant capacity and phenolic contents in fruit. Nutrition, Los Angeles, v. 21, p. 207-213, 2005.

SELLAPPAN, S.; AKOH, C. C.; KREWER, G. Phenolic compounds and antioxidant capacity of Georgia-grown blueberries and blackberries. Journal of Agricultural and Food Chemistry, Washington, v. 50, p. 2.432-2.438, 2002.

SIRIWOHARN, T.; WROLSTAD, R. E.; FINN, C. E.; PEREIRA, C. B. Influence of cultivar, maturity, and sampling on blackberry (Rubus L. Hybrids) anthocyanins, polyphenolics, and antioxidant properties. Journal of Agriculture and Food Chemistry, Washington, v. 52, p. 8.021-8.030, 2004.

SWAIN, T.; HILLIS, W. E. The phenolic constituents of Prunus domestica I.- The quantitative analysis of phenolic constituents. Journal of Science and Food Agriculture. Washington, v. 10, p. 63-68, 1959.

WANG, S. Y.; LIN, H. S. Antioxidant activity in fruits and leaves of blackberry, raspberry, and strawberry varies with cultivar and developmental stage. Journal of Agriculture and Food Chemistry, Washington, v. 48, p. 140-146, 2000.

WANG, S. Y.; MAAS, J. L.; PAYNE, J.A.;GALLETA, G. J. Ellagic acid content in small fruits mayhaws, and other plants. Journal Small Fruit and Viticulture, Louisiana, v. 2, n. 4, p. 11-49, 1994.

YING, G.; ZHAO, C. M.; JUN, W. On Rubbus resources in Human and Fujian provinces. In: INTERNATIONAL HORTICULTURAL CONGRESS, 23., 1990, Florence. Anais... p. 4014. 